TRANSACTIONS OF THE

AMERICAN MATHEMATICAL SOCIETY

Volume 360, Number 7, July 2008, Pages 3839-3856

S 0002-9947(08)04388-2

Article electronically published on February 27, 2008

\title{
THE ISOTRIVIAL CASE IN THE MORDELL-LANG THEOREM
}

\author{
DRAGOS GHIOCA
}

\begin{abstract}
We determine the structure of the intersection of a finitely generated subgroup of a semiabelian variety $G$ defined over a finite field with a closed subvariety $X \subset G$. We also study a related question in the context of a power of the additive group scheme.
\end{abstract}

\section{INTRODUCTION}

Faltings proves in 2 the Mordell-Lang conjecture in characteristic 0 , showing that the intersection of a subvariety $X$ of a semiabelian variety $G$ (defined over a field of characteristic 0) with a finitely generated subgroup $\Gamma$ of $G$ is a finite union of cosets of subgroups of $\Gamma$. In particular, this shows that an irreducible subvariety $X \subset G$ has a Zariski dense intersection with a finitely generated subgroup $\Gamma \subset G$ if and only if $X$ is a translate of an algebraic subgroup of $G$. The obvious translation of this last statement to semiabelian varieties defined over fields of positive characteristic fails to be true due to the presence of the varieties defined over finite fields. In [1, Abramovich and Voloch conjecture a function-field version of the Mordell-Lang statement in positive characteristic by formulating a condition which has to be satisfied by the subvariety $X$, if $X$ has a Zariski dense intersection with a finitely generated subgroup $\Gamma \subset G$. The conjecture is proved, using modeltheoretic techniques, by Hrushovski in [5. However, [5] does not offer a description of the intersection of $X$ with $\Gamma$. Moosa and Scanlon provide in [8 and 9] a first answer toward the last question in the isotrivial case, under an extra assumption on the subgroup $\Gamma$. In the present paper we are able to remove their assumption.

Let $G$ be a semiabelian variety defined over a finite field $\mathbb{F}_{q}$. Let $F$ be the corresponding Frobenius for $\mathbb{F}_{q}$. Then $F \in \operatorname{End}(G)$. Let $K$ be a field extension of $\mathbb{F}_{q}$. Let $X$ be a subvariety of $G$ defined over $K$ (in this paper, all subvarieties will be closed). In [8] and [9], Moosa and Scanlon discussed the intersection of the $K$ points of $X$ with a finitely generated $\mathbb{Z}[F]$-submodule $\Gamma$ of $G(K)$. They proved that the intersection is a finite union of $F$-sets in $\Gamma$ (see Definition 2.4). Our goal for this paper is to extend their result to the case when $\Gamma$ is a finitely generated subgroup of $G(K)$ (not necessarily invariant under $F$ ). In a somewhat different direction, we also show that their result remains valid when the semiabelian variety is replaced by a power of the additive group scheme. We also mention that Rahim Moosa and the author were able to describe in [4, through completely different methods, the

Received by the editors February 7, 2006 and, in revised form, July 16, 2006.

2000 Mathematics Subject Classification. Primary 11G10; Secondary 11G25.

(C)2008 American Mathematical Society 
intersection of $X$ with the divisible hull of a finitely generated $\mathbb{Z}[F]$-submodule of $G$.

In Section 2 we state our main results. In Section 3 we will prove our main theorem for semiabelian varieties, while in Section 4 we will show how our statement for $\mathbb{G}_{a}^{g}$ can be proved along similar lines as the result in 8 .

We note that our Mordell-Lang statement for the additive group scheme is actually a Mordell-Lang statement for Drinfeld modules defined over finite fields. For the general statement of the Mordell-Lang conjecture for Drinfeld modules we refer the reader to 3 .

The author thanks Rahim Moosa for his comments on an earlier version of the present paper and Thomas Scanlon for introducing the beautiful questions related to the Mordell-Lang Conjecture. The author also thanks the referee and the editor for their helpful comments.

\section{StATEMENT OF OUR MAIN RESUlts}

Everywhere in this paper, $\bar{Y}$ represents the Zariski closure of the set $Y$.

A central notion for the present paper is the notion of a Frobenius ring. This notion was first introduced by Moosa and Scanlon (see Definition 2.1 in [9]). We extend their definition to also include rings of finite characteristic.

Definition 2.1. Let $R$ be a Dedekind domain with the property that for every nonzero prime ideal $\mathfrak{p} \subset R, R / \mathfrak{p}$ is a finite field. We call $R[F]$ a Frobenius ring over $R$ if the following properties are satisfied:

(i) $R[F]$ is a finite integral extension of $R$.

(ii) $F$ is not a zero divisor in $R[F]$.

(iii) The ideal $F^{\infty} R[F]:=\bigcap_{n \geq 0} F^{n} R[F]$ is trivial.

The classical example of a Frobenius ring associated to a semiabelian variety $G$ defined over the finite field $\mathbb{F}_{q}$ is $\mathbb{Z}[F]$, where $F \in \operatorname{End}(G)$ is the endomorphism of $G$ induced by the Frobenius map on $\mathbb{F}_{q}$. This Frobenius ring is discussed in 8 and [9. Also, $\mathbb{F}_{p}[F] \subset \operatorname{End}\left(\mathbb{G}_{a}\right)$ is a Frobenius ring over itself when the distinguished element $F \in \mathbb{F}_{p}[F]$ is the Frobenius on $\mathbb{F}_{q}$. We define the notion of groupless $F$-sets contained in a module over a Frobenius ring.

Definition 2.2. Let $R[F]$ be a Frobenius ring over $R$ and let $M$ be an $R[F]$-module. For $a \in M$ and $\delta \in \mathbb{N}^{*}$, we denote the $F^{\delta}$-orbit of $a$ by $S(a ; \delta):=\left\{F^{\delta n} a \mid n \in \mathbb{N}\right\}$. If $a_{1}, \ldots, a_{k} \in M$ and $\delta_{1}, \ldots, \delta_{k} \in \mathbb{N}^{*}$, then we denote the sum of the $F^{\delta_{i}}$-orbits of $a_{i}$ by

$$
S\left(a_{1}, \ldots, a_{k} ; \delta_{1}, \ldots, \delta_{k}\right)=\left\{\sum_{i=1}^{k} F^{\delta_{i} n_{i}} a_{i} \mid\left(n_{1}, \ldots, n_{k}\right) \in \mathbb{N}^{k}\right\} .
$$

A set of the form $b+S\left(a_{1}, \ldots, a_{k} ; \delta_{1}, \ldots, \delta_{k}\right)$ with $b, a_{1}, \ldots, a_{k} \in M$ is called a groupless $F$-set based in $M$. We do allow in our definition of groupless $F$-sets $k=0$, in which case, the groupless $F$-set consists of the single point $b$. We denote by $\mathrm{GF}_{M}$ the set of all groupless $F$-sets based in $M$. For every subgroup $\Gamma \subset M$, we denote by $\mathrm{GF}_{M}(\Gamma)$ the collection of groupless $F$-sets contained in $\Gamma$ and based in $M$. When $M$ is clear from the context, we will drop the index $M$ from our notation.

Remark 2.3. Each groupless $F$-set $O$ is based in a finitely generated $R[F]$-module. 
Definition 2.4. Let $M$ be a module over a Frobenius ring $R[F]$. Let $\Gamma \subset M$ be a subgroup. A set of the form $(C+H) \subset \Gamma$, where $C \in \mathrm{GF}_{M}(\Gamma)$ and $H$ is a subgroup of $\Gamma$, is called an $F$-set in $\Gamma$ based in $M$. The collection of all such $F$-sets in $\Gamma$ is denoted by $\mathrm{F}_{M}(\Gamma)$. When $M$ is clear from the context, we will drop the index $M$ from our notation.

Let $G$ be a semiabelian variety defined over $\mathbb{F}_{q}$. Let $F$ be the Frobenius on $\mathbb{F}_{q}$. Let $K$ be a finitely generated regular extension of $\mathbb{F}_{q}$. We fix an algebraic closure $K^{\text {alg }}$ of $K$. Let $\Gamma$ be a finitely generated subgroup of $G(K)$. We denote by $\mathrm{F}(\Gamma)$ and $\operatorname{GF}(\Gamma)$ the collection of $F$-sets and, respectively, the collection of groupless $F$ sets in $\Gamma$ based in $G\left(K^{\text {alg }}\right)$ (which is obviously a $\mathbb{Z}[F]$-module). When we do not mention the $\mathbb{Z}[F]$-submodule containing the base points for the $F$-sets contained in $\Gamma$, then we will always understand that the corresponding submodule is $G\left(K^{\text {alg }}\right)$. The following theorem is our main result for semiabelian varieties.

Theorem 2.5. Let $G, K$ and $\Gamma$ be as in the above paragraph. Let $X$ be a $K$ subvariety of $G$. Then $X(K) \cap \Gamma=\bigcup_{i=1}^{r}\left(C_{i}+\Delta_{i}\right)$, where $\left(C_{i}+\Delta_{i}\right) \in \mathrm{F}(\Gamma)$. Moreover, the subgroups $\Delta_{i}$ are of the form $G_{i}(K) \cap \Gamma$, where $G_{i}$ is an algebraic subgroup of $G$ defined over $\mathbb{F}_{q}$.

As mentioned in Section 1, the result of our Theorem [2.5 was proved in [8] (see Theorem 7.8) and in $[9$ (see Theorem 2.1) for finitely generated $\mathbb{Z}[F]$-modules $\Gamma \subset G(K)$. Because $\mathbb{Z}[F]$ is a finite extension of $\mathbb{Z}$, each finitely generated $\mathbb{Z}[F]$ module is also a finitely generated group (but not every finitely generated group is invariant under $F$ ).

Now we formulate the setting for our Mordell-Lang statement for the additive group scheme. As before, $p$ is a prime number and $q$ is a power of $p$, while $F$ is the Frobenius on $\mathbb{F}_{q}$. Trivially, $\mathbb{F}_{p}[F]$ is a Frobenius ring over itself. We extend the action of $\mathbb{F}_{p}[F] \subset \operatorname{End}\left(\mathbb{G}_{a}\right)$ diagonally to any power of the additive group scheme.

Let $K$ be a field extension of $\mathbb{F}_{q}$. We fix an algebraic closure $K^{\text {alg }}$ of $K$. Let $\Gamma$ be a finitely generated $\mathbb{F}_{p}[F]$-submodule of $\mathbb{G}_{a}^{g}(K)$. We denote by $\mathrm{F}(\Gamma)$ and $\mathrm{GF}(\Gamma)$ the $F$-sets and, respectively, the groupless $F$-sets in $\Gamma$ based in the module $\mathbb{G}_{a}^{g}\left(K^{\text {alg }}\right)$ (with respect to the Frobenius ring $\mathbb{F}_{p}[F]$ ). For us, the $F$-sets in Section 4 will always be based in $\mathbb{G}_{a}^{g}\left(K^{\mathrm{alg}}\right)$.

We will explain in Section 4 that the following Mordell-Lang statement for $\mathbb{G}_{a}^{g}$ follows along the same lines as Theorem 7.8 in 8 . Moreover, our Theorem 2.6 is the analogue of Theorem 7.8 in 8 for a power of the additive group scheme. We also note that the translation of our Theorem 2.5 for the additive group scheme is trivial because any finitely generated subgroup of the additive group scheme (in characteristic $p$ ) is finite.

Theorem 2.6. Let $K$ be a finitely generated regular field extension of $\mathbb{F}_{q}$. Let $\Gamma \subset \mathbb{G}_{a}^{g}(K)$ be a finitely generated $\mathbb{F}_{p}[F]$-module. Let $X$ be an affine subvariety of $\mathbb{G}_{a}^{g}$ defined over $K$. Then $X(K) \cap \Gamma$ is a finite union of $F$-sets in $\Gamma$. Moreover, the subgroups of $\Gamma$ appearing in the intersection $X(K) \cap \Gamma$ are $\mathbb{F}_{p}[F]$-modules.

Remark 2.7. Because for any Drinfeld module $\phi$ defined over a finite field, a suitable power of the Frobenius lies in the endomorphism ring $\operatorname{End}(\phi)$ of $\phi$, Theorem 2.6 is actually a Mordell-Lang statement for Drinfeld modules defined over finite fields. This is equivalent to replacing in Theorem 7.8 from $[8$ the semiabelian variety $G$ by the power $\mathbb{G}_{a}^{g}$ of the additive group scheme together with a Drinfeld module action 
given by $\phi$, and replacing the subring $\mathbb{Z}[F] \subset \operatorname{End}(G)$ by the subring $\mathbb{F}_{p}[F] \subset$ $\operatorname{End}(\phi)$.

\section{The Mordell-Lang TheOREM For SEMiabelian VARIEties DEFINED OVER FINITE FIELDS}

Proof of Theorem 2.5. We first observe that the subgroups $\Delta_{i}$ from the intersection of $X$ with $\Gamma$ are indeed of the form $G_{i}(K) \cap \Gamma$ for algebraic groups $G_{i}$ defined over $\mathbb{F}_{q}$. Otherwise, we can always replace a subgroup $\Delta_{i}$ appearing in the intersection $X(K) \cap \Gamma$ with its Zariski closure $G_{i}$ and then intersect with $\Gamma$ (see also the proof of Lemma 7.4 in $\left[8\right.$ ). Because $G_{i}$ is the Zariski closure of a subset of $G(K)$, then $G_{i}$ is defined over $K$. Because $G_{i}$ is an algebraic subgroup of $G$, then $G_{i}$ is defined over $\mathbb{F}_{q}^{\text {alg }}$. Because $K$ is a regular extension of $\mathbb{F}_{q}$, we conclude that $G_{i}$ is defined over $\mathbb{F}_{q}=K \cap \mathbb{F}_{q}^{\text {alg }}$.

We will prove the main statement of Theorem 2.5 by induction on $\operatorname{dim}(X)$. Clearly, when $\operatorname{dim}(X)=0$ the statement holds (the intersection is a finite collection of points in this case). Assume the statement holds for $\operatorname{dim}(X)<d$ and we prove that it also holds for $\operatorname{dim}(X)=d$.

We will use in our proof a number of reduction steps.

Step 1. Because $X(K) \cap \Gamma=\overline{X(K) \cap \Gamma} \cap \Gamma$ we may assume that $X(K) \cap \Gamma$ is Zariski dense in $X$.

Step 2. At the expense of replacing $X$ by one of its irreducible components, we may assume $X$ is irreducible. Each irreducible component of $X$ has Zariski dense intersection with $\Gamma$. If Theorem 2.5 holds for each irreducible component of $X$, then it also holds for $X$.

Step 3. We may assume the stabilizer $\operatorname{Stab}_{G}(X)$ of $X$ in $G$ is trivial. Indeed, let $H:=\operatorname{Stab}_{G}(X)$. Then $H$ is defined over $K$ (because $X$ is defined over $K$ ), and also $H$ is defined over $\mathbb{F}_{q}^{\text {alg }}$ (because it is an algebraic subgroup of $G$ ). Thus $H$ is defined over $\mathbb{F}_{q}$. Let $\pi: G \rightarrow G / H$ be the natural projection. Let $\hat{G}, \hat{X}$ and $\hat{\Gamma}$ be the images of $G, X$ and $\Gamma$ through $\pi$. Then $\hat{G}$ is a semiabelian variety defined over $\mathbb{F}_{q}$, and $\hat{\Gamma}$ is a finitely generated subgroup of $\hat{G}(K)$, and $\hat{X}$ is defined over $K$. Then $\operatorname{Stab}_{\hat{G}}(\hat{X})=\{0\}$. Assume Theorem 2.5 holds for $\hat{X} \subset \hat{G}$. Then $\hat{X}(K) \cap \hat{\Gamma}$ is a finite union of $F$-sets in $\hat{\Gamma}$. Using the fact that the kernel of $\left.\pi\right|_{\Gamma}$ stabilizes $X(K) \cap \Gamma$, we conclude

$$
X(K) \cap \Gamma=\left.\pi\right|_{\Gamma} ^{-1}(\hat{X}(K) \cap \hat{\Gamma}),
$$

which shows that $X(K) \cap \Gamma$ is also a finite union of $F$-sets.

Therefore, from now on we work under the assumptions that

(i) $\overline{X(K) \cap \Gamma}=X$;

(ii) $X$ is irreducible;

(iii) $\operatorname{Stab}_{G}(X)=\{0\}$.

Let $\widetilde{\Gamma}$ be the $\mathbb{Z}[F]$-module generated by $\Gamma$. Because $\Gamma$ is finitely generated and $F$ is integral over $\mathbb{Z}$, then also $\widetilde{\Gamma}$ is finitely generated. By Theorem 7.8 of $[\underline{8}, X(K) \cap \widetilde{\Gamma}$ is a finite union of $F$-sets in $\widetilde{\Gamma}$. Moreover, there are finitely many groupless $F$-sets $C_{i}$ and $\mathbb{Z}[F]$-submodules $H_{i} \subset \widetilde{\Gamma}$ such that

$$
X(K) \cap \widetilde{\Gamma}=\bigcup_{i}\left(C_{i}+H_{i}\right)
$$


We want to show $\bigcup_{i}\left(C_{i}+H_{i}\right) \cap \Gamma$ is a finite union of $F$-sets in $\Gamma$. It suffices to show that for each $i$, there exists a finite union $B_{i}$ of $F$-sets in $\Gamma$ such that $\left(C_{i}+H_{i}\right) \cap \Gamma \subset B_{i} \subset X(K)$. Indeed, the existence of such $B_{i}$ yields

$$
X(K) \cap \Gamma=\bigcup_{i} B_{i}
$$

as desired.

Case 1. $\operatorname{dim} \overline{C_{i}+H_{i}}<d$.

Let $X_{i}:=\overline{C_{i}+H_{i}}$. Then $X_{i}$ is defined over $K$ because $\left(C_{i}+H_{i}\right) \subset \widetilde{\Gamma} \subset G(K)$. Also $\operatorname{dim}\left(X_{i}\right)<d$. So, by the induction hypothesis, $B_{i}:=X_{i}(K) \cap \Gamma$ is a finite union of $F$-sets in $\Gamma$. Clearly, $\left(C_{i}+H_{i}\right) \cap \Gamma \subset B_{i} \subset X(K)$, because $X_{i} \subset X$.

Case 2. $\operatorname{dim} \overline{C_{i}+H_{i}}=d$.

Because $X=\overline{X(K) \cap \Gamma}$, then $X=\overline{X(K) \cap \widetilde{\Gamma}}$. Moreover, $X$ is irreducible and so, because $\operatorname{dim} \overline{C_{i}+H_{i}}=\operatorname{dim}(X)$, then $X=\overline{C_{i}+H_{i}}$. Hence $H_{i} \subset \operatorname{Stab}_{G}(X)$ because

$$
C_{i}+H_{i}+H_{i}=C_{i}+H_{i} \text {, and so } \overline{C_{i}+H_{i}}+H_{i} \subset \overline{C_{i}+H_{i}} .
$$

Because $\operatorname{Stab}_{G}(X)$ is trivial, we conclude $H_{i}=\{0\}$, and so $C_{i}+H_{i}=C_{i}$. We let $B_{i}:=C_{i} \cap \Gamma$. The following Theorem 3.1 will conclude the proof of Theorem2.5.

Theorem 3.1. Let $M$ be a finitely generated $\mathbb{Z}[F]$-submodule of $G\left(K^{\mathrm{alg}}\right)$ and let $O \in \mathrm{GF}_{M}$. If $\Gamma$ is a finitely generated subgroup of $G\left(K^{\mathrm{alg}}\right)$, then $O \cap \Gamma$ is a finite union of groupless $F$-sets based in $M$.

Proof. Let

$$
f(X):=X^{g}-\sum_{i=0}^{g-1} \alpha_{i} X^{i}
$$

be the minimal polynomial for $F$ over $\mathbb{Z}$ (i.e. $f(F)=0$ in $\operatorname{End}(G)$ ). Let $r_{1}, \ldots, r_{g}$ be all the roots in $\mathbb{C}$ of $f(X)$.

Claim 3.2. All roots $r_{i}$ of $f$ are distinct complex numbers, greater than 1 in absolute value.

Proof of Claim 3.2. It suffices to prove the minimal polynomial $h(X)$ of a power $F^{n}$ of $F$ has distinct roots of absolute value greater than 1 . Indeed, because $f$ is the minimal polynomial for $F$ over $\mathbb{Z}$ in $\operatorname{End}(G)$, then $f(X) \mid h\left(X^{n}\right)$. Assuming $h(X)$ has distinct roots, then $h\left(X^{n}\right)$ also has distinct roots, and so $f(X)$ has distinct roots. The claim about the absolute values of $r_{i}$ follows immediately if we know this to be true for the absolute values of the roots of $h$.

Let $0 \rightarrow T \rightarrow G \rightarrow A \rightarrow 0$ be a short exact sequence of group varieties, with $T$ being a torus and $A$ an abelian variety defined over a finite field. Moreover, according to Proposition 9.1 in [7, $A$ is isogenous with a direct product of simple abelian varieties defined over $\mathbb{F}_{p}^{\text {alg: }}$

$$
A \sim A_{0}:=\prod_{i=1}^{l} A_{i}^{n_{i}},
$$

where $n_{i} \geq 1$ and the abelian varieties $A_{i} \mp A_{j}$ for $i \neq j$.

Let $n$ be a positive integer number such that the above exact sequence of algebraic groups is defined over $\mathbb{F}_{q^{n}}$, the isogeny between $A$ and $A_{0}$ is defined over 
$\mathbb{F}_{q^{n}}$, and each $A_{i}$ is defined over $\mathbb{F}_{q^{n}}$. It suffices to prove Claim 3.2 for the minimal polynomial $h$ of $F^{n} \in \operatorname{End}(G)$ over $\mathbb{Z}$.

Because there are no nonzero group homomorphisms between $T$ and $A, \operatorname{End}(G)$ embeds into $\operatorname{End}(T) \times \operatorname{End}(A)$. Hence, $h$ is the least common multiple of the minimal polynomial of $F^{n}$ on $T$ and the minimal polynomial $h_{0}$ for the induced Frobenius $F^{n}$ on $A$. Actually, the minimal polynomial for $F^{n}$ on $T$ is $X-q^{n}$, while the roots of $h_{0}$ are all in absolute value $\sqrt{q^{n}}$ (see Theorem 14.1 in [7]). Thus all roots of $h$ are bigger than 1 in absolute value. It remains to show that all the roots of $h_{0}$ are simple.

Because $A$ is isogenous to $A_{0}$, then the Frobenius $F^{n}$ has the same monic polynomial $h_{0}$ over $\mathbb{Z}$ in $\operatorname{End}\left(A_{0}\right)$. The Frobenius $F^{n}$ restricts to an endomorphism of each copy of $A_{i}$ in $A_{i}^{n_{i}}$. Because $A_{i}$ is a simple abelian variety, $\operatorname{End}\left(A_{i}\right)$ is a domain, and so, the minimal polynomial $h_{i}$ of $F^{n} \in \operatorname{End}\left(A_{i}\right)$ over $\mathbb{Z}$ is an irreducible polynomial. Moreover, $h_{i} \mid h_{0}$ because they both kill the Frobenius $F^{n}$ on $A_{i}$, but $h_{i}$ is the minimal polynomial of the Frobenius $F^{n}$ on $A_{i}$. The least common multiple $h_{0}^{\prime}$ of all $h_{i}$ satisfies $\operatorname{ker}\left(h_{0}^{\prime}\left(F^{n}\right)\right)=A_{0}$, and so $h_{0}^{\prime}\left(F^{n}\right)=0$. Therefore, $h_{0} \mid h_{0}^{\prime}$, and so $h_{0}=h_{0}^{\prime}$. Thus we proved that each $h_{i}$ has distinct roots, and so their least common multiple $h_{0}$ has distinct roots.

The definition of $f$ shows that for every point $P \in G\left(K^{\text {alg }}\right)$,

$$
F^{g} P=\sum_{j=0}^{g-1} \alpha_{j} F^{j} P .
$$

We conclude that for all $n \geq g$,

$$
F^{n} P=\sum_{j=0}^{g-1} \alpha_{j} F^{n-g+j} P .
$$

For each $j \in\{0, \ldots, g-1\}$ we define the sequence $\left\{z_{j, n}\right\}_{n \geq 0}$ as follows:

$$
\begin{gathered}
z_{j, n}=0 \text { if } 0 \leq n \leq g-1 \text { and } n \neq j \\
z_{j, j}=1 \text { and } \\
z_{j, n}=\sum_{l=0}^{g-1} \alpha_{l} z_{j, n-g+l} \text { for all } n \geq g .
\end{gathered}
$$

Using (4) and (5) we obtain that

$$
F^{n} P=\sum_{j=0}^{g-1} z_{j, n} F^{j} P, \text { for every } 0 \leq n \leq g-1 .
$$

Using (3), (6) and (7), an easy induction on $n$ shows that

$$
F^{n} P=\sum_{j=0}^{g-1} z_{j, n} F^{j} P, \text { for every } n \geq 0 .
$$

For each $j,\left\{z_{j, n}\right\}_{n}$ is a recursively defined sequence. Moreover, using Claim 3.2. the roots of the characteristic polynomial

$$
X^{g}-\sum_{i=0}^{g-1} \alpha_{i} X^{i}
$$


associated to each such recursively defined sequence are nonzero, distinct complex numbers. We will prove later in Lemma 3.9 that for each $j \in\{0, \ldots, g-1\}$ there exist $\left\{\gamma_{j, l}\right\}_{1 \leq l \leq g} \subset \mathbb{Q}^{\text {alg }}$ such that for every $n \in \mathbb{N}$,

$$
z_{j, n}=\sum_{1 \leq l \leq g} \gamma_{j, l} r_{l}^{n}
$$

Equations (8) and (10) show that for every $n$ and for every $P \in G\left(K^{\text {alg }}\right)$,

$$
F^{n} P=\sum_{0 \leq j \leq g-1}\left(\sum_{1 \leq l \leq g} \gamma_{j, l} r_{l}^{n}\right) F^{j} P \text {. }
$$

If $O \cap \Gamma$ is finite, then we are done. So, from now on, we may assume $O \cap \Gamma$ is infinite. Also, we may and do assume $\Gamma \subset M$ (otherwise we replace $\Gamma$ with $\Gamma \cap M$ ).

Let $O:=Q+S\left(P_{1}, \ldots, P_{k} ; \delta_{1}, \ldots, \delta_{k}\right)$, where $Q, P_{1}, \ldots, P_{k} \in M$ and $\delta_{i} \in \mathbb{N}^{*}$ for every $i \in\{1, \ldots, k\}$. We may assume that $\delta_{1}=\cdots=\delta_{k}=1$, in which case we let

$$
S\left(P_{1}, \ldots, P_{k} ; 1\right):=S\left(P_{1}, \ldots, P_{k} ; \delta_{1}, \ldots, \delta_{k}\right)
$$

Indeed, if we show that $\left(Q+S\left(P_{1}, \ldots, P_{k} ; 1\right)\right) \cap \Gamma$ is a union of groupless $F$-sets, then also its subsequent intersection with $\left(Q+S\left(P_{1}, \ldots, P_{k} ; \delta_{1}, \ldots, \delta_{k}\right)\right)$ is a finite union of groupless $F$-sets, as shown in part $(a)$ of Lemma 3.7 in 8 .

Because $M$ is a finitely generated $\mathbb{Z}$-module, $M$ is isomorphic with a direct sum of its finite torsion submodule $M_{\text {tor }}$ and a free $\mathbb{Z}$-submodule $M_{1}$.

For each $i \in\{1, \ldots, k\}$ and for each $j \in\{0, \ldots, g-1\}$, let $F^{j} P_{i}:=T_{i}^{(j)}+Q_{i}^{(j)}$, with $T_{i}^{(j)} \in M_{\text {tor }}$ and $Q_{i}^{(j)} \in M_{1}$. Also, let $Q:=T_{0}+Q_{0}$, where $T_{0} \in M_{\text {tor }}$ and $Q_{0} \in M_{1}$.

Let $R_{1}, \ldots, R_{m}$ be a basis for the $\mathbb{Z}$-module $M_{1}$. For each $j \in\{0, \ldots, g-1\}$ and for each $i \in\{1, \ldots, k\}$, let

$$
Q_{i}^{(j)}:=\sum_{l=1}^{m} a_{i, j}^{(l)} R_{l}
$$

where $a_{i, j}^{(l)} \in \mathbb{Z}$. Finally, let $a_{0}^{(1)}, \ldots, a_{0}^{(m)} \in \mathbb{Z}$ such that $Q_{0}=\sum_{j=1}^{m} a_{0}^{(j)} R_{j}$.

For every $n \in \mathbb{N}$ and for every $i \in\{1, \ldots, k\}$, (18) and the definitions of $Q_{i}^{(j)}$ and $T_{i}^{(j)}$ yield

$$
F^{n} P_{i}=\sum_{0 \leq j \leq g-1} z_{j, n}\left(T_{i}^{(j)}+Q_{i}^{(j)}\right)=\sum_{0 \leq j \leq g-1} z_{j, n} T_{i}^{(j)}+\sum_{0 \leq j \leq g-1} z_{j, n} Q_{i}^{(j)} .
$$

Because $T_{i}^{(j)} \in M_{\mathrm{tor}}$, then for each $\left(n_{1}, \ldots, n_{k}\right) \in \mathbb{N}^{k}$,

$$
T_{0}+\sum_{i=1}^{k} \sum_{j=0}^{g-1} z_{j, n_{i}} T_{i}^{(j)} \in M_{\mathrm{tor}}
$$

Also, because $Q_{0}$ and all $Q_{i}^{(j)}$ are in $M_{1}$ and because $z_{j, n} \in \mathbb{Z}$, then for each $\left(n_{1}, \ldots, n_{k}\right) \in \mathbb{N}^{k}$,

$$
Q_{0}+\sum_{i=1}^{k} \sum_{j=0}^{g-1} z_{j, n_{i}} Q_{i}^{(j)} \in M_{1}
$$


Moreover,

$$
Q+\sum_{i=1}^{k} F^{n_{i}} P_{i}=\left(T_{0}+\sum_{\substack{1 \leq i \leq k \\ 0 \leq j \leq g-1}} z_{j, n_{i}} T_{i}^{(j)}\right)+\left(Q_{0}+\sum_{\substack{1 \leq i \leq k \\ 0 \leq j \leq g-1}} z_{j, n_{i}} Q_{i}^{(j)}\right)
$$

For each $h \in M_{\text {tor }}$, if $\left(h+M_{1}\right) \cap \Gamma$ is not empty, we fix $\left(h+U_{h}\right) \in \Gamma$ for some $U_{h} \in M_{1}$. Let $\Gamma_{1}:=\Gamma \cap M_{1}$. Then

$$
\left(h+M_{1}\right) \cap \Gamma=h+U_{h}+\Gamma_{1} .
$$

For each $h \in M_{\text {tor }}$, we let $O_{h}:=\left\{P \in O \mid P=h+P^{\prime}\right.$ with $\left.P^{\prime} \in M_{1}\right\}$. Then using (15), we get

$$
O \cap \Gamma=\bigcup_{h \in M_{\text {tor }}} O_{h} \cap\left(h+U_{h}+\Gamma_{1}\right)=\bigcup_{h \in M_{\text {tor }}}\left(h+\left(\left(-h+O_{h}\right) \cap\left(U_{h}+\Gamma_{1}\right)\right)\right)
$$

We observe that the rank of $\Gamma_{1}$ as a $\mathbb{Z}$-module is strictly positive because otherwise, as shown by (16), $O \cap \Gamma$ is finite (and we assumed it is infinite).

Therefore (16) and (14) yield $O \cap \Gamma$ is a (finite) union over all $h \in M_{\text {tor }}$ of the points $\left(Q+\sum_{i} F^{n_{i}} P_{i}\right) \in O$ corresponding to tuples $\left(n_{1}, \ldots, n_{k}\right)$ such that

$$
T_{0}+\sum_{i, j} z_{j, n_{i}} T_{i}^{(j)}=h \text { and }\left(Q_{0}+\sum_{i, j} z_{j, n_{i}} Q_{i}^{(j)}\right) \in\left(U_{h}+\Gamma_{1}\right) .
$$

Claim 3.3. Let $h \in M_{\text {tor }}$ be fixed. The set of tuples $\left(n_{1}, \ldots, n_{k}\right) \in \mathbb{N}^{k}$ for which

$$
h=T_{0}+\sum_{i, j} z_{j, n_{i}} T_{i}^{(j)}
$$

is a finite union of cosets of semigroups of $\mathbb{N}^{k}$ (a semigroup of $\mathbb{N}^{k}$ is the intersection with $\mathbb{N}^{k}$ of a subgroup of $\mathbb{Z}^{k}$; a coset of a semigroup is the intersection with $\mathbb{N}^{k}$ of a coset of a subgroup of $\mathbb{Z}^{k}$ ).

Proof of Claim 3.3. Indeed, let $N \in \mathbb{N}^{*}$ such that $M_{\text {tor }} \subset G[N]$. Because for each $j \in\{0, \ldots, g-1\}, z_{j, n}$ is a recursively defined sequence over $\mathbb{Z}$ (as shown by (4), (5) and ([6) ), then the sequence $\left\{z_{j, n}\right\}_{n}$ is preperiodic modulo $N$ (a recursively defined sequence is preperiodic modulo any integral modulus). We recall that a sequence $\left\{x_{n}\right\}_{n}$ is preperiodic if there exists a positive integer $l$ such that the subsequence $\left\{x_{n}\right\}_{n \geq l}$ is periodic.

Now, because of our choice for $N, N T_{i}^{(j)}=0$ for each $i$ and $j$. Thus $\left\{z_{j, n} T_{i}^{(j)}\right\}_{n}$ is preperiodic for every $j$ and $i$. So, each value taken by $T_{0}+\sum_{i, j} z_{j, n_{i}} T_{i}^{(j)}$ is attained for tuples $\left(n_{1}, \ldots, n_{k}\right)$ which belong to a finite union of cosets of semigroups of $\mathbb{N}^{k}$.

Claim 3.4. Let $h \in M_{\text {tor }}$ be fixed such that $\left(h+M_{1}\right) \cap \Gamma$ is not empty. The tuples $\left(n_{1}, \ldots, n_{k}\right)$ for which

$$
\left(Q_{0}+\sum_{i, j} z_{j, n_{i}} Q_{i}^{(j)}\right) \in\left(U_{h}+\Gamma_{1}\right)
$$

form a finite union of cosets of semigroups of $\mathbb{N}^{k}$. 
Before proving Claim 3.4 we observe that its proof will finish the proof of our Theorem 3.1. The intersection of two finite unions of cosets of semigroups of $\mathbb{N}^{k}$ is a finite union of cosets of semigroups of $\mathbb{N}^{k}$. Using the results of Claim 3.3 and Claim 3.4, we get that for each fixed $h \in M_{\text {tor }}$, there is a finite union $W_{h}$ of cosets of semigroups of $\mathbb{N}^{k}$ such that a tuple $\left(n_{1}, \ldots, n_{k}\right) \in \mathbb{N}^{k}$ satisfies

$$
\begin{gathered}
h=T_{0}+\sum_{i, j} z_{j, n_{i}} T_{i}^{(j)} \text { and } \\
\left(Q_{0}+\sum_{i, j} z_{j, n_{i}} Q_{i}^{(j)}\right) \in\left(U_{h}+\Gamma_{1}\right),
\end{gathered}
$$

if and only if $\left(n_{1}, \ldots, n_{k}\right) \in W_{h}$. Using (16), (17) and that $\left|M_{\text {tor }}\right|<\aleph_{0}$, we conclude that the tuples $\left(n_{1}, \ldots, n_{k}\right)$ for which

$$
Q+\sum_{i=1}^{k} F^{n_{i}} P_{i} \in \Gamma
$$

form a finite union of cosets of semigroups of $\mathbb{N}^{k}$. Lemma 3.4 of [8] shows that the set of points $\left(Q+\sum_{i} F^{n_{i}} P_{i}\right) \in O$ corresponding to tuples $\left(n_{1}, \ldots, n_{k}\right)$ contained in a finite union of cosets of semigroups of $\mathbb{N}^{k}$ is a finite union of groupless $F$-sets.

Proof of Claim 3.4. We first define three classes of subsets of $\mathbb{Z}^{m}$.

Definition 3.5. A C-subset of $\mathbb{Z}^{m}$ is a set $C\left(d_{1}, \ldots, d_{m}, D_{1}, D_{2}\right)$, where $d_{1}, \ldots, d_{m}$, $D_{1}, D_{2} \in \mathbb{Z}$ and $D_{2} \neq 0$, containing all solutions $\left(x_{1}, \ldots, x_{m}\right) \in \mathbb{Z}^{m}$ of $\sum_{i=1}^{m} d_{i} x_{i} \equiv$ $D_{1}\left(\bmod D_{2}\right)$.

An L-subset of $\mathbb{Z}^{m}$ is a set $L\left(d_{1}, \ldots, d_{m}, D\right)$, where $d_{1}, \ldots, d_{m}, D \in \mathbb{Z}$, containing all solutions $\left(x_{1}, \ldots, x_{m}\right) \in \mathbb{Z}^{m}$ of $\sum_{i=1}^{m} d_{i} x_{i}=D$.

A CL-subset of $\mathbb{Z}^{m}$ is either a C-subset or an L-subset of $\mathbb{Z}^{m}$.

Subclaim 3.6. There exist CL-subsets $S_{1}, \ldots, S_{m}$ of $\mathbb{Z}^{m}$ such that a point $P:=$ $\sum_{j=1}^{m} c^{(j)} R_{j}$ lies in $U_{h}+\Gamma_{1}$ if and only if

$$
\left(c^{(1)}, \ldots, c^{(m)}\right) \in \bigcap_{i=1}^{m} S_{i} .
$$

Proof of Subclaim 3.6. Because $\Gamma_{1} \subset M_{1}$ and $M_{1}$ is a free $\mathbb{Z}$-module with basis $\left\{R_{1}, \ldots, R_{m}\right\}$, we can find (after a possible relabelling of $R_{1}, \ldots, R_{m}$ ) a $\mathbb{Z}$-basis $V_{1}, \ldots, V_{n}(1 \leq n \leq m)$ of $\Gamma_{1}$ of the following form:

$$
\begin{aligned}
& V_{1}=\beta_{1}^{\left(i_{1}\right)} R_{i_{1}}+\cdots+\beta_{1}^{(m)} R_{m} \\
& V_{2}=\beta_{2}^{\left(i_{2}\right)} R_{i_{2}}+\cdots+\beta_{2}^{(m)} R_{m}
\end{aligned}
$$

and in general, $V_{j}=\beta_{j}^{\left(i_{j}\right)} R_{i_{j}}+\cdots+\beta_{j}^{(m)} R_{m}$ for each $j \leq n$, where

$$
1 \leq i_{1}<i_{2}<\cdots<i_{n} \leq m
$$

and all $\beta_{j}^{(i)} \in \mathbb{Z}$. We also assume $\beta_{j}^{\left(i_{j}\right)} \neq 0$ for every $j \in\{1, \ldots, n\}$.

Let $b_{0}^{(1)}, \ldots, b_{0}^{(m)} \in \mathbb{Z}$ such that $U_{h}=\sum_{j=1}^{m} b_{0}^{(j)} R_{j}$. Then $P \in\left(U_{h}+\Gamma_{1}\right)$ if and only if there exist integers $k_{1}, \ldots, k_{n}$ such that

$$
P=U_{h}+\sum_{i=1}^{n} k_{i} V_{i} \text {. }
$$


Using the expressions of the $V_{i}, U_{h}$ and $P$ in terms of the $\mathbb{Z}$-basis $\left\{R_{1}, \ldots, R_{m}\right\}$ of $M_{1}$, we obtain the following relations for the coefficients $c^{(j)}$ :

$$
\begin{gathered}
c^{(j)}=b_{0}^{(j)} \text { for every } 1 \leq j<i_{1} ; \\
c^{(j)}=b_{0}^{(j)}+k_{1} \beta_{1}^{(j)} \text { for every } i_{1} \leq j<i_{2} ; \\
c^{(j)}=b_{0}^{(j)}+k_{1} \beta_{1}^{(j)}+k_{2} \beta_{2}^{(j)} \text { for every } i_{2} \leq j<i_{3}
\end{gathered}
$$

and so on, until

$$
c^{(m)}=b_{0}^{(m)}+\sum_{i=1}^{n} k_{i} \beta_{i}^{(m)} .
$$

We interpret the above relations as follows: the numbers $c^{(i)}$ are the unknowns, while the numbers $k_{1}, \ldots, k_{n}$ are integer parameters, and all $b_{0}^{(i)}$ and $\beta_{j}^{(i)}$ are integer constants. We will show, by eliminating the parameters $k_{i}$, that the unknowns $c^{(j)}$ must satisfy $n$ linear congruences and $(m-n)$ linear equations with coefficients involving only the $b_{0}^{(i)}$ and the $\beta_{j}^{(i)}$. Each such equation will generate a CL-set.

Therefore we express equation (24) for $j=i_{1}$ as a linear congruence modulo $\beta_{1}^{\left(i_{1}\right)}$ (by eliminating $k_{1}$ ) and obtain

$$
c^{\left(i_{1}\right)} \equiv b_{0}^{\left(i_{1}\right)}\left(\bmod \beta_{1}^{\left(i_{1}\right)}\right) .
$$

Also from (24) for $j=i_{1}$, we get $k_{1}=\frac{c^{\left(i_{1}\right)}-b_{0}^{\left(i_{1}\right)}}{\beta_{1}^{\left(i_{1}\right)}}$. Then we substitute this formula for $k_{1}$ in (24) for all $i_{1}<j<i_{2}$ and obtain

$$
c^{(j)}=b_{0}^{(j)}+\frac{c^{\left(i_{1}\right)}-b_{0}^{\left(i_{1}\right)}}{\beta_{1}^{\left(i_{1}\right)}} \beta_{1}^{(j)} \text { for every } i_{1}<j<i_{2} .
$$

Then we express (25) for $j=i_{2}$ as a linear congruence modulo $\beta_{2}^{\left(i_{2}\right)}$ (also using the expression for $k_{1}$ computed above). We obtain

$$
c^{\left(i_{2}\right)} \equiv b_{0}^{\left(i_{2}\right)}+\frac{c^{\left(i_{1}\right)}-b_{0}^{\left(i_{1}\right)}}{\beta_{1}^{\left(i_{1}\right)}} \beta_{1}^{\left(i_{2}\right)}\left(\bmod \beta_{2}^{\left(i_{2}\right)}\right) .
$$

Next we equate $k_{2}$ from (25) for $j=i_{2}$ (also using the above formula for $k_{1}$ ) and obtain

$$
k_{2}=\frac{c^{\left(i_{2}\right)}-b_{0}^{\left(i_{2}\right)}-\frac{c^{\left(i_{1}\right)}-b_{0}^{\left(i_{1}\right)}}{\beta_{1}^{\left(i_{1}\right)}} \beta_{1}^{\left(i_{2}\right)}}{\beta_{2}^{\left(i_{2}\right)}} .
$$

Then we substitute this formula for $k_{2}$ in (25) for $i_{2}<j<i_{3}$ and obtain

$$
c^{(j)}=b_{0}^{(j)}+\frac{c^{\left(i_{1}\right)}-b_{0}^{\left(i_{1}\right)}}{\beta_{1}^{\left(i_{1}\right)}} \cdot \beta_{1}^{(j)}+\frac{c^{\left(i_{2}\right)}-b_{0}^{\left(i_{2}\right)}-\frac{c^{\left(i_{1}\right)}-b_{0}^{\left(i_{1}\right)}}{\beta_{1}^{\left(i_{1}\right)}} \beta_{1}^{\left(i_{2}\right)}}{\beta_{2}^{\left(i_{2}\right)}} \cdot \beta_{2}^{(j)} .
$$

We go on as above until we express $c^{(m)}$ in terms of $b_{0}^{(m)}, c^{\left(i_{1}\right)}, \ldots, c^{\left(i_{n}\right)}$ and $\left\{\beta_{j}^{(l)}\right\}_{j, l}$. We observe that all of the above congruences and linear equations can be written as linear congruences or linear equations over $\mathbb{Z}$ (after clearing the denominators). 
For example, the congruence equation (29) can be written as the following linear congruence over $\mathbb{Z}$ :

$$
\beta_{1}^{\left(i_{1}\right)} \cdot c^{\left(i_{2}\right)} \equiv \beta_{1}^{\left(i_{1}\right)} b_{0}^{\left(i_{2}\right)}+\left(c^{\left(i_{1}\right)}-b_{0}^{\left(i_{1}\right)}\right) \beta_{1}^{\left(i_{2}\right)}\left(\bmod \beta_{1}^{\left(i_{1}\right)} \cdot \beta_{2}^{\left(i_{2}\right)}\right) .
$$

Hence all the above conditions that need be satisfied by $c^{(j)}$ such that

$$
\sum_{j=1}^{m} c^{(j)} R_{j} \in\left(U_{h}+\Gamma_{1}\right)
$$

are either linear equations or linear congruences with integer coefficients. There are precisely $n$ congruences (corresponding to the $n$ degrees of freedom introduced by the parameters $\left.k_{i}\right)$ and $(m-n)$ linear equations.

We will show that for each $S_{i}$, there exists a finite number of cosets of semigroups $W_{j}^{(i)} \subset \mathbb{N}^{k}$ such that $\left(n_{1}, \ldots, n_{k}\right) \in \bigcup_{j} W_{j}^{(i)}$ if and only if $\left(c^{(1)}, \ldots, c^{(m)}\right) \in S_{i}$, where

$$
Q_{0}+\sum_{i, j} z_{j, n_{i}} Q_{i}^{(j)}=: \sum_{j=1}^{m} c^{(j)} R_{j}
$$

This will show that there exists a finite number of cosets of semigroups

$$
\widetilde{W}:=\bigcap_{i=1}^{m}\left(\bigcup_{j} W_{j}^{(i)}\right) \subset \mathbb{N}^{k}
$$

such that $\left(n_{1}, \ldots, n_{k}\right) \in \widetilde{W}$ if and only if

$$
\left(Q_{0}+\sum_{i, j} z_{j, n_{i}} Q_{i}^{(j)}\right) \in\left(U_{h}+\Gamma_{1}\right) .
$$

Subclaim 3.7. Let $C:=C\left(d_{1}, \ldots, d_{m}, D_{1}, D_{2}\right)$ be a C-subset of $\mathbb{Z}^{m}$. There exists a finite number of cosets of semigroups $W_{j} \subset \mathbb{N}^{k}$ such that $\left(n_{1}, \ldots, n_{k}\right) \in \bigcup_{j} W_{j}$ if and only if $\left(c^{(1)}, \ldots, c^{(m)}\right) \in C$, where

$$
Q_{0}+\sum_{i, j} z_{j, n_{i}} Q_{i}^{(j)}=: \sum_{j=1}^{m} c^{(j)} R_{j} .
$$

Proof of Subclaim 3.7. Using (12) (and the similar expression for $Q_{0}$ in terms of $\left.R_{1}, \ldots, R_{m}\right)$, we conclude that for every $1 \leq j \leq m$ :

$$
c^{(j)}=a_{0}^{(j)}+\sum_{\substack{1 \leq i \leq k \\ 0 \leq l \leq g-1}} a_{i, l}^{(j)} z_{l, n_{i}} .
$$

Hence, the congruence equation $\sum_{j=1}^{m} d_{j} c^{(j)} \equiv D_{1}\left(\bmod D_{2}\right)$ yields the following congruence:

$$
\sum_{j=0}^{g-1} \sum_{i=1}^{k} d_{j, i} z_{j, n_{i}} \equiv D_{1}^{\prime}\left(\bmod D_{2}\right)
$$

for some integer numbers $D_{1}^{\prime}$ and $\left\{d_{j, i}\right\}_{j, i}$ (we recall that all $a_{0}^{(j)} \in \mathbb{Z}$ and all $\left.a_{i, l}^{(j)} \in \mathbb{Z}\right)$. Recursively defined sequences such as $\left\{z_{j, n}\right\}_{n}$ are preperiodic modulo any nonzero integer (hence, they are preperiodic modulo $D_{2}$ ). Therefore all solutions 
$\left(n_{1}, \ldots, n_{k}\right) \in \mathbb{N}^{k}$ to (33) belong to a finite union $\bigcup_{j} W_{j}$ of cosets of semigroups of $\mathbb{N}^{k}$.

Subclaim 3.8. Let $L:=L\left(d_{1}, \ldots, d_{m}, D\right)$ be an L-subset of $\mathbb{Z}^{m}$. There exists a finite number of cosets of semigroups $W_{j} \subset \mathbb{N}^{k}$ such that $\left(n_{1}, \ldots, n_{k}\right) \in \bigcup_{j} W_{j}$ if and only if $\left(c^{(1)}, \ldots, c^{(m)}\right) \in L$, where

$$
Q_{0}+\sum_{i, j} z_{j, n_{i}} Q_{i}^{(j)}=: \sum_{j=1}^{m} c^{(j)} R_{j} .
$$

Proof of Subclaim 3.8. Using (12) and (10), we conclude that for every $1 \leq j \leq m$ :

$$
c^{(j)}=a_{0}^{(j)}+\sum_{\substack{1 \leq i \leq k \\ 0 \leq l \leq g-1}} a_{i, l}^{(j)} \sum_{e=1}^{g} \gamma_{l, e} r_{e}^{n_{i}} .
$$

The linear equation $\sum_{j=1}^{m} d_{j} c^{(j)}=D$ yields the following equation (after collecting the coefficients of $r_{e}^{n_{i}}$ for each $1 \leq e \leq g$ and each $\left.1 \leq i \leq k\right)$ :

$$
\sum_{e=1}^{g} \sum_{i=1}^{k} d_{e, i} r_{e}^{n_{i}}=D_{0}
$$

where all $d_{e, i}$ and $D_{0}$ are algebraic numbers (actually, $D_{0} \in \mathbb{Z}$, but we will not need to use this). A tuple $\left(n_{1}, \ldots, n_{k}\right) \in \mathbb{N}^{k}$ satisfying (35) corresponds to an intersection point of the linear variety $L_{0}$ in $\left(\mathbb{G}_{m}^{g}\right)^{k}\left(\mathbb{Q}^{\text {alg }}\right)$ given by the equation

$$
\sum_{e=1}^{g} \sum_{i=1}^{k} d_{e, i} X_{e, i}=D_{0}
$$

with the finitely generated subgroup $G_{0}$ of $\left(\mathbb{G}_{m}^{g}\right)^{k}\left(\mathbb{Q}^{\text {alg }}\right)$ spanned by

$$
\begin{aligned}
v_{1}:=\left(r_{1}, \ldots, r_{g}, 1, \ldots, 1\right) ; v_{2}:=\left(1, \ldots, 1, r_{1}, \ldots, r_{g}, 1, \ldots, 1\right) ; \ldots ; \\
v_{k}:=\left(1, \ldots, 1, r_{1}, \ldots, r_{g}\right) .
\end{aligned}
$$

Each vector $v_{i}$ has $g k$ components. The correspondence between the tuples $\left(n_{1}, \ldots, n_{k}\right)$ satisfying (35) and the solutions of (36) is given by

$$
\left(n_{1}, \ldots, n_{k}\right) \rightarrow v_{1}^{n_{1}} v_{2}^{n_{2}} \ldots v_{k}^{n_{k}} .
$$

In (37) there are $k$ multiplicatively independent generators for $G_{0}$ (we are using the fact that $\left|r_{i}\right| \neq 1$, for each $i$ ). Hence $G_{0} \simeq \mathbb{Z}^{k}$, where the isomorphism $\sigma: G_{0} \rightarrow$ $\mathbb{Z}^{k}$ is given on the $\mathbb{Z}$-basis $\left\{v_{1}, \ldots, v_{k}\right\}$ of $G_{0}$ by sending the vector $v_{i} \in G_{0}$ to the vector $(0, \ldots, 1, \ldots, 0) \in \mathbb{Z}^{k}$, whose only nonzero entry is on the $i^{\text {th }}$ coordinate.

By the Lang Theorem for $\mathbb{G}_{m}^{g k}$ (see [6]), we conclude the intersection of $L_{0}\left(\mathbb{Q}^{\text {alg }}\right.$ ) and $G_{0}$ is a finite union of cosets of subgroups of $G_{0}$. The cosets of subgroups of $G_{0}$ correspond through $\sigma$ to cosets of subgroups of $\mathbb{Z}^{k}$ (for all possible tuples $\left(n_{1}, \ldots, n_{k}\right) \in \mathbb{Z}^{k}$ satisfying (35) ). Hence the tuples $\left(n_{1}, \ldots, n_{k}\right) \in \mathbb{N}^{k}$ which satisfy (35) belong to a finite union of cosets of semigroups of $\mathbb{N}^{k}$.

Because the intersection of two cosets of semigroups of $\mathbb{N}^{k}$ is a finite union of cosets of semigroups, Subclaims 3.7 and 3.8 finish the proof of Claim 3.4 .

As explained before the proof of Claim 3.4, the results of Claims 3.3 and 3.4 finish the proof of Theorem 3.1 . 
The following classical result on recursively defined sequences was used in the proof of Theorem 2.5. We provide its proof for completeness.

Lemma 3.9. Let $g \in \mathbb{N}^{*}$ and let $\alpha_{0}, \ldots, \alpha_{g-1} \in \mathbb{C}$. Assume the roots $\left\{r_{j}\right\}_{j=1}^{g}$ of the polynomial

$$
f(X):=X^{g}-\sum_{i=0}^{g-1} \alpha_{i} X^{i}
$$

are distinct, nonzero complex numbers. Let $\left\{z_{n}\right\}_{n \geq 0}$ be the recursively defined sequence given by

$$
z_{n}=\sum_{i=0}^{g-1} \alpha_{i} z_{n-g+i} \text { for all } n \geq g .
$$

Therefore, $z_{0}, \ldots, z_{g-1}$ are some arbitrary complex numbers, while all other elements of the sequence $\left\{z_{n}\right\}$ are uniquely determined for $n \geq g$ from the above recursively defined relation. Then there exist $\left\{\gamma_{j}\right\}_{j=1}^{g} \subset \mathbb{C}$ such that for every $n \geq 0$

$$
z_{n}=\sum_{j=1}^{g} \gamma_{j} r_{j}^{n}
$$

Proof. Because the numbers $r_{j}$ are nonzero, distinct complex numbers, then the following system of $g$ equations with $g$ unknowns $\gamma_{j}$ has a unique solution (the associated determinant is a nonzero Vandermonde determinant):

$$
\sum_{j=1}^{g} \gamma_{j} r_{j}^{i}=z_{i} \text { for } i \in\{0, \ldots, g-1\} .
$$

Moreover, if all $z_{i} \in \mathbb{Q}^{\text {alg }}$ for $i \in\{0, \ldots, g-1\}$ and all $r_{j} \in \mathbb{Q}^{\text {alg }}$ for $j \in\{1, \ldots, g\}$, then also all $\gamma_{j} \in \mathbb{Q}^{\text {alg }}$ for $j \in\{1, \ldots, g\}$.

Because each $r_{j}$ is a root of $f(X)$, then the sequence $\left\{z_{n}^{\prime}\right\}_{n} \subset \mathbb{C}$ defined by

$$
z_{n}^{\prime}=\sum_{j=1}^{g} \gamma_{j} r_{j}^{n}
$$

satisfies the same recursively defined relation as the one satisfied by the sequence $\left\{z_{n}\right\}_{n}$. Moreover, by (38), $z_{n}^{\prime}=z_{n}$ for all $n \in\{0, \ldots, g-1\}$. Because both sequences $\left\{z_{n}^{\prime}\right\}_{n}$ and $\left\{z_{n}\right\}_{n}$ are uniquely determined by their first $g$ elements and the recursive relation they both satisfy, we conclude $z_{n}^{\prime}=z_{n}$. This concludes the proof of Lemma 3.9.

\section{A Mordell-Lang statement for the AdDitive Group SCHEME}

In this section, $F$ is the Frobenius corresponding to $\mathbb{F}_{q}$. Also, $g$ is a positive integer. We will deduce Theorem 2.6 from the following more general result.

Theorem 4.1. Let $K$ be a finitely generated regular extension of $\mathbb{F}_{q}$. Then for each algebraic subgroup $H \subset \mathbb{G}_{a}^{g}$ defined over $\mathbb{F}_{q}$ and for every variety $X \subset \mathbb{G}_{a}^{g} / H$ defined over $K$ and for every finitely generated $\mathbb{F}_{p}[F]$-submodule $\Gamma \subset\left(\mathbb{G}_{a}^{g} / H\right)(K)$, the intersection $X(K) \cap \Gamma$ is a finite union of $F$-sets in $\Gamma$ based in $\left(\mathbb{G}_{a}^{g} / H\right)\left(K^{\text {alg }}\right)$. Moreover, the subgroups of $\Gamma$ appearing in the intersection $X(K) \cap \Gamma$ are $\mathbb{F}_{p}[F]$ modules. 
We first observe that because the algebraic group $H$ in Theorem 4.1 is defined over $\mathbb{F}_{q}$, then $\mathbb{G}_{a}^{g} / H$ is invariant under $F$, and so it makes sense to talk about $\mathbb{F}_{p}[F]$-submodules of $\left(\mathbb{G}_{a}^{g} / H\right)(K)$. Theorem 2.6 is a particular case of Theorem 4.1 for $H=\{0\}$.

We will prove Theorem 4.1 through a series of lemmas. Our argument follows along the line of ideas present in the proof of Theorem 7.8 in [8].

We fix an algebraic group $U$ which is the quotient of $\mathbb{G}_{a}^{g}$ through an algebraic subgroup $H$ defined over the finite field $\mathbb{F}_{q}$. The following result (which is the equivalent for $\mathbb{G}_{a}^{g}$ of Lemma 7.5 in [8]) will be used in the proof of our Theorem 4.1.

Lemma 4.2. Let $K$ be a finitely generated field extension of $\mathbb{F}_{q}$ and let $\Gamma \subset \mathbb{G}_{a}^{g}(K)$ be a finitely generated $\mathbb{F}_{p}[F]$-submodule.

(a) The F-divisible hull $\widetilde{\Gamma}$ of $\Gamma$ in $\mathbb{G}_{a}^{g}(K)$, i.e. the set of all $x \in \mathbb{G}_{a}^{g}(K)$ such that $F^{m} x \in \Gamma$ for some $m \geq 0$, is a finitely generated $\mathbb{F}_{p}[F]$-module.

(b) Let $\Gamma^{\prime}$ be the image of $\Gamma$ through the canonical projection $\sigma: \mathbb{G}_{a}^{g} \rightarrow U$. Let $\widetilde{\Gamma^{\prime}}$ be the $F$-divisible hull of $\Gamma^{\prime}$ in $U(K)$. Then $\widetilde{\Gamma^{\prime}}$ is a finitely generated $\mathbb{F}_{p}[F]$-module.

(c) For each $m>0$, both $\Gamma / F^{m} \Gamma$ and $\Gamma^{\prime} / F^{m} \Gamma^{\prime}$ are finite.

(d) For each $m>0$, there exists $m_{1} \geq 0$ such that $\Gamma^{\prime} \backslash F^{m} \Gamma^{\prime} \subset U(K) \backslash U\left(K^{q^{m_{1}}}\right)$.

Proof. (a) First we observe that the $F$-divisible hull $\widetilde{\Gamma}$ of $\Gamma$ is an $\mathbb{F}_{p}[F]$-module. Indeed, if $x \in \widetilde{\Gamma}$ and $m \in \mathbb{N}$ such that $F^{m} x \in \Gamma$, then for every $f \in \mathbb{F}_{p}[F]$,

$$
F^{m}(f(x))=f\left(F^{m} x\right) \in f(\Gamma) \subset \Gamma .
$$

Therefore $f(x) \in \widetilde{\Gamma}$, showing that $\widetilde{\Gamma}$ is an $\mathbb{F}_{p}[F]$-module.

It suffices to prove $(a)$ under the extra assumption that $\Gamma=\Gamma_{0}^{g}$ (the cartesian product of $\Gamma_{0}$ with itself $g$ times), where $\Gamma_{0} \subset K$ is a finitely generated $\mathbb{F}_{p}[F]$ module. Indeed, let $\Gamma_{0}$ be the finitely generated $\mathbb{F}_{p}[F]$-submodule of $K$ spanned by all the generators (over $\mathbb{F}_{p}[F]$ ) of the projections of $\Gamma$ on the $g$ coordinates of $\mathbb{G}_{a}^{g}(K)$. Clearly $\Gamma \subset \Gamma_{0}^{g}$ and $\widetilde{\Gamma_{0}^{g}}=\left(\widetilde{\Gamma_{0}}\right)^{g}$. So, if we prove $(a)$ for $\Gamma_{0}$, then the result of $(a)$ holds also for $\Gamma_{0}^{g}$ and implicitly for its submodule $\Gamma$ (the $F$-divisible hull $\widetilde{\Gamma}$ of $\Gamma$ is an $\mathbb{F}_{p}[F]$-submodule of the $F$-divisible hull $\widetilde{\Gamma_{0}^{g}}$ of $\Gamma_{0}^{g}$, and a submodule of a finitely generated module is also finitely generated). So, we are left to show that the $F$-divisible hull $\widetilde{\Gamma_{0}}$ of $\Gamma_{0}$ in $K$ is a finitely generated $\mathbb{F}_{p}[F]$-module. This follows from Lemma 4 of [10] applied to the Drinfeld module $\phi$ defined on $\mathbb{F}_{p}[t]$ by $\phi_{t}=F$. However, for the sake of completeness, we provide a direct proof which does not use the theory of Drinfeld modules. We will use the following Claim 4.3 to finish the proof of $(a)$.

Claim 4.3. Let $R$ be a Dedekind domain such that for every proper ideal $I \subset R$, $R / I$ is finite. Let $M$ be a finite rank $R$-module (i.e. $\operatorname{dim}_{\operatorname{Frac}(R)} M \otimes_{R} \operatorname{Frac}(R)$ is finite). Assume there exists a function $h: M \rightarrow \mathbb{R}_{\geq 0}$ satisfying the following properties:

(i) (Triangle inequality) $h(x \pm y) \leq h(x)+h(y)$, for every $x, y \in M$.

(ii) For every $D>0$, there exist only finitely many $x \in M$ such that $h(x) \leq D$.

(iii) There exists $a \in R \backslash\{0\}$ such that $R / a R$ is finite and for all $x \in M$, $h(a x) \geq 4 h(x)$.

Then $M$ is finitely generated. 
Before proving Claim 4.3 we show that its statement yields that $\widetilde{\Gamma_{0}}$ is a finitely generated $\mathbb{F}_{p}[F]$-module. Indeed, we use Claim 4.3 with $R=\mathbb{F}_{p}[F], M=\widetilde{\Gamma_{0}}$, $a=F^{2}$, and $h$ being the usual logarithmic Weil height on $K$ (restricted to $\widetilde{\Gamma_{0}}$ ) corresponding to the set of valuations associated to the irreducible divisors of a projective, normal variety $V$ defined over a finite field and whose function field is $K$ (see [11 for the construction of the Weil height on any finitely generated field $K)$. Clearly, $\mathbb{F}_{p}[F]$ is a Dedekind domain with the property from Claim 4.3 .

The $\mathbb{F}_{p}[F]$-module $\widetilde{\Gamma_{0}}$ lies in the divisible hull of the finitely generated module $\Gamma_{0}$, and so it has finite rank over $\mathbb{F}_{p}[F]$. Clearly $h$ satisfies the triangle inequality. Condition (ii) in Claim 4.3 is simply the Northcott Theorem for the Weil height $h$ on the finitely generated field $K$. As for condition (iii), clearly $\mathbb{F}_{p}[F] /\left(F^{2}\right)$ is finite. On the other hand, for every $x \in K, h\left(F^{2} x\right)=q^{2} h(x) \geq 4 h(x)$, as desired in condition (iii) of Claim 4.3. Thus the conditions of Claim 4.3 are satisfied and its conclusion shows that $\widetilde{\Gamma_{0}}$ is finitely generated.

Proof of Claim 4.3, Let $a \in R$ as in condition (iii). We first show $M_{\text {tor }}$ is finite. Indeed, let $x \in M_{\text {tor }}$, and let $I \subset R$ be a proper ideal of $R$ such that $I x=0$. Then because $R / I$ is finite, there exist positive integers $m<n$ such that $a^{n}-a^{m} \in I$. Using condition (iii), we conclude:

$$
h\left(a^{n} x\right) \geq 4^{n-m} h\left(a^{m} x\right) .
$$

Because $a^{n} x=a^{m} x$, we get $h\left(a^{n} x\right)=h\left(a^{m} x\right)=0$. Another application of condition (iii) yields

$$
0=h\left(a^{n} x\right) \geq 4^{n} h(x) \geq 0 .
$$

Hence we proved that for every torsion point $x \in M, h(x)=0$. Then using condition (ii), we conclude $M_{\text {tor }}$ is finite, as desired. By Lemma 3 of [10, because $M_{\text {tor }}$ is finite, and $M$ has finite rank and $R$ satisfies the hypothesis of Claim 4.3. we conclude $M / a M$ is finite.

From this point, our proof follows the classical descent argument in the MordellWeil theorem (see [11]). Let $y_{1}, \ldots, y_{k}$ be coset representatives for $a M$ in $M$. Define then

$$
B=\max _{i \in\{1, \ldots, k\}} h\left(y_{i}\right) .
$$

Consider the set $Z=\{x \in M \mid h(x) \leq B\}$, which is finite according to condition (ii). Let $N$ be the finitely generated $R$-submodule of $M$ which is spanned by $Z$.

We claim that $M=N$. If we suppose this is not the case, then by condition (ii) we can pick $y \in M-N$ which minimizes $h(y)$. Because $N$ contains all the coset representatives of $a M$ in $M$, we can find $i \in\{1, \ldots, k\}$ such that $y-y_{i} \in a M$. Let $x \in M$ be such that $y-y_{i}=a x$. Then $x \notin N$ because otherwise it would follow that $y \in N$ (we already know $y_{i} \in N$ ). By our choice of $y$ and by properties (iii) and $(i)$, we have

$$
h(y) \leq h(x) \leq \frac{h\left(y-y_{i}\right)}{4} \leq \frac{h(y)+h\left(y_{i}\right)}{4} \leq \frac{h(y)+B}{4} .
$$

This means that $h(y) \leq \frac{B}{3}<B$. This contradicts the fact that $y \notin N$ because $N$ contains all the elements $z \in M$ such that $h(z) \leq B$. This contradiction shows that indeed $M=N$, and so $M$ is finitely generated. 
(b) It suffices to show that there exists a finitely generated $\mathbb{F}_{p}[F]$-module $M \subset$ $\mathbb{G}_{a}^{g}(K)$ such that the $F$-divisible hull of $(\Gamma+H(K))$ in $\mathbb{G}_{a}^{g}(K)$ equals $(M+H(K))$. Then $\widetilde{\Gamma^{\prime}}=\sigma(M)$ is finitely generated as an $\mathbb{F}_{p}[F]$-module.

Because $H(K) \cap \mathbb{G}_{a}^{g}\left(K^{q}\right)=H\left(K^{q}\right)$, we conclude $H(K) / H\left(K^{q}\right)$ is a subgroup of

$$
\mathbb{G}_{a}^{g}(K) / \mathbb{G}_{a}^{g}\left(K^{q}\right) \stackrel{\sim}{\rightarrow}\left(K / K^{q}\right)^{g} .
$$

Because $K$ is finitely generated over $\mathbb{F}_{q}$, we conclude $K / K^{q}$ is finite. Thus $\mathbb{G}_{a}^{g}(K) / \mathbb{G}_{a}^{g}\left(K^{q}\right)$ and $H(K) / H\left(K^{q}\right)$ are also finite. Let $\left\{h_{1}, \ldots, h_{l}\right\}$ be coset representatives for $H\left(K^{q}\right)$ in $H(K)$. Because $H$ is defined over $\mathbb{F}_{q}$, then $H\left(K^{q}\right)=$ $F(H(K))$. Thus we obtain that for every $m \in \mathbb{N},\left\{F^{m} h_{1}, \ldots, F^{m} h_{l}\right\}$ are coset representatives for $F^{m+1}(H(K))=H\left(K^{q^{m+1}}\right)$ in $F^{m}(H(K))=H\left(K^{q^{m}}\right)$. Moreover,

$$
\left\{\sum_{i=0}^{m-1} F^{i} h_{f(i)} \mid f:\{0, \ldots, m-1\} \rightarrow\{1, \ldots, l\}\right\}
$$

is a complete system of coset representatives for $H\left(K^{q^{m}}\right)$ in $H(K)$. Let $M_{0}$ be the finitely generated $\mathbb{F}_{p}[F]$-module generated by the elements of $\Gamma \cup\left\{h_{1}, \ldots, h_{l}\right\}$. Let $M$ be the $F$-divisible hull of $M_{0}$ in $\mathbb{G}_{a}^{g}(K)$. By part $(a), M$ is a finitely generated $\mathbb{F}_{p}[F]$-module. We will prove that the $F$-divisible hull of $(\Gamma+H(K))$ in $\mathbb{G}_{a}^{g}(K)$ equals $(M+H(K))$.

By the construction of $M$, clearly $M$ and $H(K)$ are subsets of the $F$-divisible hull of $(\Gamma+H(K))$ in $\mathbb{G}_{a}^{g}(K)$. Therefore $(M+H(K))$ is contained in the $F$-divisible hull of $(\Gamma+H(K))$ in $\mathbb{G}_{a}^{g}(K)$.

Let $x \in \mathbb{G}_{a}^{g}(K)$ and assume there exists $m \in \mathbb{N}^{*}$ such that $F^{m} x \in(\Gamma+H(K))$. So, $F^{m} x=\gamma+h$, where $\gamma \in \Gamma$ and $h \in H(K)$. We write $h=h_{0}+F^{m} h_{1}$, where $h_{0}$ is a coset representative from (39) of $H\left(K^{q^{m}}\right)=F^{m}(H(K))$ in $H(K)$ and $h_{1} \in H(K)$. Hence

$$
F^{m}\left(x-h_{1}\right)=\gamma+h_{0} \in M_{0} .
$$

Thus $\left(x-h_{1}\right) \in M$, and so $x \in(M+H(K))$, as desired. This concludes the proof of $(b)$.

(c) Because $\Gamma$ and $\Gamma^{\prime}$ are finitely generated $\mathbb{F}_{p}[F]$-modules, then $\Gamma / F^{m} \Gamma$ and $\Gamma^{\prime} / F^{m} \Gamma^{\prime}$ are finitely generated $\left(\mathbb{F}_{p}[F] / F^{m} \mathbb{F}_{p}[F]\right)$-modules. But $\mathbb{F}_{p}[F] / F^{m} \mathbb{F}_{p}[F]$ is a finite ring. Hence $\Gamma / F^{m} \Gamma$ and $\Gamma^{\prime} / F^{m} \Gamma^{\prime}$ are finite, as desired.

(d) Because the $F$-divisible hull $\widetilde{\Gamma}^{\prime}$ of $\Gamma^{\prime}$ in $U(K)$ is finitely generated as an $\mathbb{F}_{p}[F]$-module, then there exists $m_{0}>0$ such that $F^{m_{0}} \widetilde{\Gamma^{\prime}} \subset \Gamma^{\prime}$. Let $m_{1}:=m_{0}+m$. Then

$$
\Gamma^{\prime} \cap U\left(K^{q^{m_{1}}}\right) \subset F^{m_{1}} \widetilde{\Gamma^{\prime}} \subset F^{m} \Gamma^{\prime} .
$$

Hence $\Gamma^{\prime} \backslash F^{m} \Gamma^{\prime} \subset U(K) \backslash U\left(K^{q^{m_{1}}}\right)$.

The proofs of the next two lemmas are identical with the proofs of Corollary 7.3 and, respectively, Lemma 3.9 in 8 .

Lemma 4.4. Let $K$ be a finitely generated regular field extension of $\mathbb{F}_{q}$. Let $\Gamma \subset$ $U(K)$ be a finitely generated $\mathbb{F}_{p}[F]$-module. Let $B$ be a finite union of sets of the form $(C+\Delta) \subset \Gamma$, where $C \in \operatorname{GF}(\Gamma)$ and $\Delta$ is a subgroup of $\Gamma$ invariant under $F$. Let $X \subset U$ be a subvariety defined over $K$. Let $\Sigma:=\bigcup_{n \geq 0} F^{n} B$ and suppose that $\Sigma \subset X(K)$. Then there exists a finite union $V$ of sets of the form 
$\left(C^{\prime}+\Delta^{\prime}\right) \subset \Gamma$, where $C^{\prime} \in \mathrm{GF}(\Gamma)$ and $\Delta^{\prime}$ is a subgroup of $\Gamma$ invariant under $F$, such that $\Sigma \subset V \subset X(K)$.

Lemma 4.5. Suppose $M$ is a finitely generated $\mathbb{F}_{p}[F]$-module. Let $B$ and $V$ be two finite unions of $F$-sets in $M$. In addition, assume the groups appearing in $B$ and $V$ are invariant under some power $F^{b}$ of $F$. Then $B \cap V$ is a finite union of $F$-sets in $M$; moreover, the groups appearing in $B \cap V$ are invariant under $F^{b}$.

Now we can prove Theorem 4.1 .

Proof of Theorem 4.1. Our proof follows the proof of Theorem 7.8 in 8 . We proceed by induction on $\operatorname{dim}(X)$. If $\operatorname{dim}(X)=0$, then $X(K) \cap \Gamma$ is a finite union of points and, clearly, the conclusion of Theorem 4.1 holds. We assume Theorem 4.1 holds for $\operatorname{dim}(X)<d$ (for some $d \geq 1$ ) and we will prove that it also holds for $\operatorname{dim}(X)=d$.

We may assume $\overline{X(K) \cap \Gamma}=X$ (otherwise, we may replace $X$ with $\overline{X(K) \cap \Gamma}$ ). Also, we may assume $X$ is irreducible because it suffices to prove Theorem 4.1 for each irreducible component of $X$.

The next lemma shows that a translate of $X$ is defined over a finite field. The proof of Lemma 4.6 is identical with the proof of Lemma 7.7 in 8 . Everywhere in the proof of Lemma 7.7 in 8 we replace the Frobenius ring $\mathbb{Z}[F]$ with the Frobenius ring $\mathbb{F}_{p}[F]$. The only property of the Frobenius ring used in the proof of Lemma 7.7 in 8 is property $(c)$ from Lemma 4.2 and the only property of the ambient algebraic group $G$ (a semiabelian variety in 8 and $U=\mathbb{G}_{a}^{g} / H$ for us) used in the proof of Lemma 7.7 in $[8]$ is that $\bigcap_{n \geq 1} F^{n} G\left(K^{\text {sep }}\right)=G\left(\mathbb{F}_{q}^{\text {alg }}\right)$.

Lemma 4.6. Suppose $\Gamma$ is a finitely generated $\mathbb{F}_{p}[F]$-submodule of $U(K)$ and $X \subset$ $U$ is a variety defined over $K$ such that $X(K) \cap \Gamma$ is Zariski dense in $X$. Then for some $\gamma \in K^{\text {alg }},(\gamma+X)$ is defined over $\mathbb{F}_{q}^{\text {alg }}$.

Next we show that we may assume $X$ is defined over $\mathbb{F}_{q}$. Lemma 4.6 shows that there exists $\gamma \in K^{\text {alg }}$ such that $(\gamma+X)$ is defined over $\mathbb{F}_{q}^{\text {alg }}$. Let $\Gamma^{\prime}$ be the finitely generated $\mathbb{F}_{p}[F]$-module generated by $\gamma$ and the elements of $\Gamma$. Let $K^{\prime}:=K(\gamma)$. Because $X$ is defined over $K^{\prime}$ and $\gamma \in K^{\prime}$, then $(\gamma+X)$ is defined over $K^{\prime}$. But we already know that $(\gamma+X)$ is defined over $\mathbb{F}_{q}^{\text {alg }}$. Hence $Y:=(\gamma+X)$ is defined over $\mathbb{F}_{q^{b}}:=K^{\prime} \cap \mathbb{F}_{q}^{\text {alg }}$ (we recall that $K \cap \mathbb{F}_{q}^{\text {alg }}=\mathbb{F}_{q}$ ). Assuming Theorem 4.1 holds for $Y\left(K^{\prime}\right) \cap \Gamma^{\prime}$ (note that $\Gamma^{\prime}$ is also a finitely generated $\mathbb{F}_{p}\left[F^{b}\right]$-module), we obtain that

$$
X\left(K^{\prime}\right) \cap \Gamma^{\prime}=-\gamma+\left(Y\left(K^{\prime}\right) \cap \Gamma^{\prime}\right)
$$

is a finite union of $F$-sets in $\Gamma^{\prime}$ of the form $\left(C_{i}+\Delta_{i}\right)$, where $\Delta_{i}$ is invariant under $F^{b}$. Because $\Gamma$ is a submodule of $\Gamma^{\prime}$, we conclude

$$
X(K) \cap \Gamma=X\left(K^{\prime}\right) \cap \Gamma=\left(X\left(K^{\prime}\right) \cap \Gamma^{\prime}\right) \cap \Gamma .
$$

Hence, using Lemma 4.5 $X(K) \cap \Gamma$ is itself a finite union of $F$-sets in $\Gamma$ of the form $\left(C_{i}^{\prime}+\Delta_{i}^{\prime}\right)$, where $\Delta_{i}^{\prime}$ are $\mathbb{F}_{p}\left[F^{b}\right]$-submodules of $\Gamma$. By replacing $\Delta_{i}^{\prime}$ with the Zariski closure of $\Delta_{i}^{\prime}$ intersected with $\Gamma$ (see the proof of Lemma 7.4 in [8]), $X(K) \cap \Gamma$ is a finite union of $F$-sets in $\Gamma$ of the form $\left(C_{i}^{\prime}+\Delta_{i}^{\prime \prime}\right)$, where $\Delta_{i}^{\prime \prime}$ are $\mathbb{F}_{p}[F]$-submodules of $\Gamma$, as desired.

Therefore we reduced Theorem 4.1 to the case when $X$ is defined over $\mathbb{F}_{q}$. We may assume $\operatorname{Stab}(X) \subset U$ is trivial. Indeed, let $H_{1}=\operatorname{Stab}(X)$. Then $H_{1}$ is defined over $\mathbb{F}_{q}$, because $X$ is defined over $\mathbb{F}_{q}$. We consider the canonical quotient map 
$\pi: U \rightarrow U / H_{1}$. Clearly, $U / H_{1}$ is another quotient of $\mathbb{G}_{a}^{g}$ by an algebraic subgroup defined over $\mathbb{F}_{q}$. Let $\hat{X}$ and $\hat{\Gamma}$ be the images of $X$ and $\Gamma$ through $\pi$. Clearly $\operatorname{Stab}(\hat{X})=\{0\}$. Moreover, if $\hat{X}(K) \cap \hat{\Gamma}$ is a finite union of $F$-sets (for which the groups are actually $\mathbb{F}_{p}[F]$-submodules in $\left.\hat{\Gamma}\right)$, then

$$
X(K) \cap \Gamma=\left.\pi\right|_{\Gamma} ^{-1}(\hat{X}(K) \cap \hat{\Gamma})
$$

is also a finite union of $F$-sets for which the groups are $\mathbb{F}_{p}[F]$-submodules in $\Gamma$ (we use the fact that $\operatorname{ker}\left(\left.\pi\right|_{\Gamma}\right)=\Gamma \cap H_{1}(K)$ is a subgroup of $\Gamma$ invariant under $F$ ).

From this point on, the proof continues precisely as the proof of Theorem $7.8 \mathrm{in}$ 8. We provided in Lemmas 4.2, 4.4 and 4.5 the technical ingredients which are used in the argument from the proof of Theorem 7.8 in 8 .

The following result follows from Theorem 3.1 in [9] the same way our Theorems 2.6 and 4.1 followed from Theorem 7.8 in 8 .

Theorem 4.7. Let $K$ be an algebraically closed field extension of $\mathbb{F}_{q}$. Let $X \subset \mathbb{G}_{a}^{g}$ (for some $g \geq 1$ ) be an affine variety defined over $K$. Let $\Gamma \subset \mathbb{G}_{a}^{g}(K)$ be a finitely generated $\mathbb{F}_{p}[F]$-module. Let $\Gamma^{\prime}:=\Gamma+\mathbb{G}_{a}^{g}\left(\mathbb{F}_{q}^{\text {alg }}\right)$. Then $X(K) \cap \Gamma^{\prime}$ is a finite union of sets of the form $\left(V+Y\left(\mathbb{F}_{q}^{a l g}\right)\right)$, where $V \subset \Gamma^{\prime}$ is an $F$-set and $Y \subset \mathbb{G}_{a}^{g}$ is an affine variety defined over $\mathbb{F}_{q}^{\text {alg }}$.

\section{REFERENCES}

[1] D. Abramovich and J. F. Voloch, Toward a proof of the Mordell-Lang conjecture in characteristic $p$. Internat. Math. Res. Notices 5 (1992), 103-115. MR1162230 (94f:11051)

[2] G. Faltings, The general case of S. Lang's conjecture. Barsotti Symposium in Algebraic Geometry (Abano Terme, 1991), 175-182, Perspect. Math., 15, Academic Press, San Diego, CA, 1994.

[3] D. Ghioca, The Mordell-Lang Theorem for Drinfeld modules. Internat. Math. Res. Notices, 53 (2005), 3273-3307. MR2196099 (2006k:11105)

[4] D. Ghioca and R. Moosa, Division points on subvarieties of isotrivial semiabelian varieties, Internat. Math. Res. Notices 19 (2006), 1-23. MR.2264715 (2008c:14058)

[5] E. Hrushovski, The Mordell-Lang conjecture for function fields. J.Amer.Math.Soc 9 (1996), no.3, 667-690. MR1333294 (97h:11154)

[6] M. Laurent, Equations diophantiennes exponentielles. Inventiones Math. 78 (1984), 299-327. MR767195 (86j:11062)

[7] J. Milne, Abelian varieties. course notes available online at http://www.jmilne.org/math/.

[8] R. Moosa and T. Scanlon, F-structures and integral points on semiabelian varieties over finite fields. Amer. Journal of Math. 126 (2004), 473-522. MR2058382 (2006f:11071)

[9] R. Moosa and T. Scanlon, The Mordell-Lang Conjecture in positive characteristic revisited. Model Theory and Applications (eds. L. Bélair, P. D'Aquino, D. Marker, M. Otero, F. Point, \& A. Wilkie), 2003, 273-296. MR2159720(2007a:11085)

[10] B. Poonen, Local height functions and the Mordell-Weil theorem for Drinfeld modules, Compositio Math. 97 (1995), 349-368. MR 1353279 (96k:11075)

[11] J.-P. Serre, Lectures on the Mordell-Weil theorem. Translated from the French and edited by Martin Brown from notes by Michel Waldschmidt. Aspects of Mathematics, E15. Friedr. Vieweg \& Sohn, Braunschweig, 1989. x+218 pp. MR1002324 (90e:11086)

Department of Mathematics \& Statistics, Hamilton Hall, Room 218, McMaster University, 1280 Main Street West, Hamilton, Ontario, Canada L8S 4K1

E-mail address: dghioca@math.mcmaster.ca

Current address: Department of Mathematics and Computer Science, University of Lethbridge, Lethbridge, Alberta, Canada T1K 3M4 